\title{
COMPARAÇÃO DE DIFERENTES TÉCNICAS DE MOLDAGEM COM SILICONE DE ADIÇÃO - ESTUDO IN VITRO
}

\section{COMPARISON OF DIFFERENT IMPRESSION TECHNIQUES WITH ADDITION SILICONE - IN VITRO STUDY}

\author{
Mariana Borges Barros LIMA'; Ana Paula Rodrigues de MAGALHÃES²; Terence Romano TEIXEIRA3; Pedro Alves de LIMA3; \\ Wellington Ferreira MARTINS3; Rafael Almeida DECURCIO4; Paula de Carvalho CARDOSO² \\ 1 - Especialista em Dentística Restauradora, Curso de Especialização em Dentística Restauradora, Associação Brasileira de Odontologia - Secção \\ Goiás, Goiânia-GO, Brasil. \\ 2 - Professora Doutora, Curso de Especialização em Dentística Restauradora, Associação Brasileira de Odontologia - Secção Goiás, Goiânia-GO, Brasil. \\ 3 - Professor especialista, Curso de Especialização em Dentística Restauradora, Associação Brasileira de Odontologia - Secção Goiás, Goiânia-GO, Brasil. \\ 4 - Professor Mestre, Curso de Especialização em Dentística Restauradora, Associação Brasileira de Odontologia - Secção Goiás, Goiânia-GO, Brasil.
}

\section{RESUMO}

Objetivo: Comparar diferentes técnicas de moldagem com silicone de adição, quanto à precisão e fidelidade da cópia através de medidas manuais e sobreposição em CAD/CAM. Material e método: Foram realizadas 4 moldagens com silicone de adição de um mesmo modelo nas seguintes técnicas: T1 - dois passos com recorte; T2 - dois passos com alívio de filme PVC; T3 - dois passos sem alívio e T4 - simultânea. A fidelidade das 4 técnicas de moldagem foi avaliada por meio de mensurações de 5 dimensões nos modelos de gesso vazados em cada um dos moldes obtidos. Outra forma de análise foi a sobreposição de imagens no CAD/CAM, com a finalidade de verificar se há semelhanças ou diferenças dimensionais entre o modelo piloto e as amostras. As medidas obtidas foram submetidas ao teste ANOVA com comparações múltiplas pelo teste de Tukey $(\alpha=0,05)$. Resultados: De acordo com as mensurações, a técnica que apresentou maior discordância do modelo piloto foi a T4, não havendo diferença entre as outras técnicas. Na avaliação em CAD/CAM, as técnicas T4 e T3 apresentaram maiores diferenças em relação ao modelo original de acordo com as imagens sobrepostas. Conclusões: Todas as técnicas estudadas apresentaram alterações dimensionais, em especial a técnica simultânea e a sem alívio, tanto nas medidas obtidas quanto na análise em CAD/CAM.

Palavras-chave: Materiais para Moldagem Odontológica, Técnica de Moldagem Odontológica, Elastômeros de Silicone, Modelos Anatômicos.

\section{INTRODUÇÃO}

Na década de 1950, os elastômeros foram introduzidos na Odontologia, e podem ser classificados com silicone de adição, silicone de condensação, poliéter e polissufeto ${ }^{1}$. De 1950 até hoje os materiais evoluíram muito quanto sua composição e propriedades, além do avanço nas técnicas de moldagem² ${ }^{2}$.

O sucesso das restaurações depende de vários fatores, como a indicação correta para o caso, qualidade do preparo, a cimentação, ajustes e polimento das peças ${ }^{3}$. A etapa de moldagem é crucial para o sucesso do caso, levando-se em consideração que a precisão do modelo é alcançada com a fidelidade obtida durante o processo de moldagem. Os detalhes e características existentes no dente preparado, nos dentes vizinhos e no antagonista devem estar presentes na moldagem para que isso seja reproduzido no modelo $^{4-6}$, minimizando os erros nas peças protéticas.

Cada um dos materiais de moldagem tem suas características específicas. O polissulfeto, por exemplo, por ter água em sua reação de condensação, ele a perde para o meio, o que resulta na contração do material. O silicone por condensação também apresenta uma alteração dimensional evidente por dois motivos: devido à perda de álcool etílico por evaporação após a polimerização e a uma alta taxa de contração linear. O poliéter não apresenta nenhum subproduto na sua reação de polimerização, portanto o intervalo de tempo até vazar o molde pode ser estendido, porém por ser um produto com características hidrófilas, pode absorver água ou fluidos do meio, e por isso o molde deve ser armazenado em ambiente seco e fresco. O silicone por adição, composto por polivinilsiloxano (PVS), é um material de maior estabilidade dimensional, pois em sua reação de polimerização não ocorre a liberação de nenhum subproduto ${ }^{2,7-9}$.

O PVS possui em sua composição grupos terminais de etileno ou vinil que, quando se ligam aos grupamentos de hidretos, mediados por um catalisador de sal de platina formam a reação de presa ${ }^{1}$. Apresenta ainda uma reação secundária entre a umidade e os hidretos residuais, essa reação libera gás hidrogênio, apesar dessa liberação, o material de moldagem não apresenta nenhuma distorção ${ }^{10,11}$. Apesar de não haver alteração dimensional após sua reação de polimerização, os modelos devem ser vazados após 30 minutos, esse cuidado inibe a formação de bolhas na superfície do gesso, que pode ser causada pela liberação de hidrogênio, além de permitir a sua recuperação elástica ${ }^{8}$.

O PVS apresenta certas características tais como: estabilidade dimensional, baixa fluidez e tempo de ajuste curto. Apresentam uma resistência de ruptura de moderada a alta, devido sua 
fluidez, podem ser inseridos onde o operador preferir, possuem cores contrastantes para visualização do término do preparo e hidrofilia, evitando que os fluidos do sulco interfiram na moldagem ${ }^{3,12,13}$.

O objetivo da moldagem é manter inalteradas e precisas as informações obtidas do preparo. O PVS é o material mais usado na Odontologia para moldagem de próteses que requerem uma impressão mais precisa como coroas, facetas, onlays, inlays e próteses sobre implante ${ }^{14}$.

Interessados em minimizar as distorções apresentadas pelos materiais de moldagem, os fabricantes têm investido em melhorar suas características, trabalhando mais na sua precisão, fornecendo uma impressão mais detalhada, minimizando alguns desafios encontrados no dia a dia do consultório como a formação de bolhas, rasgamentos e repuxo ${ }^{14}$. As propriedades de fluidez do material foram alteradas para que o material tenha uma melhor adaptação aos tecidos moles e duros, melhorando a impressão $0^{2,8,13}$.

Deve-se levar em consideração algumas variáveis que podem influenciar diretamente na qualidade da moldagem tais como: a técnica empregada, a experiência do operador, material usado, manipulação correta do material, saúde gengival, controle de umidade e manejo dos tecidos moles. A espessura do material deve ser idealmente de no mínimo $2 \mathrm{~mm}$ e a moldeira deve ser rígida, para suportar as forças aplicadas durante a moldagem evitando assim que ocorram distorções. Todas essas características minimizam os erros, obtendo-se uma moldagem mais precisa e previsível ${ }^{12,15}$

Apesar das novas tecnologias e inúmeros estudos relacionados aos materiais de moldagem e às técnicas utilizadas, ainda são encontradas inexatidões do ajuste das peças protéticas, o que pode estar relacionado não só ao material, mas principalmente ao operador e à técnica empregada ${ }^{16}$. Na literatura, há poucas informações quanto à melhor técnica de moldagem com silicone de adição $0^{7,14,17}$. Por isso, o objetivo deste estudo é comparar diferentes técnicas de moldagem com silicone de adição, quanto à precisão e fidelidade da cópia através de medidas manuais e sobreposição em CAD/CAM (Computer-Aided Design/Computer-Aided Manufacturing).

\section{MATERIAIS E MÉTODOS}

Baseado em estudos anteriores, o material de escolha para a pesquisa foi o silicone de adição, composto por PVS (Elite HD+, Zermack, Badia Polesine, Itália). O modelo piloto correspondia a uma arcada parcial de um manequim para trabalhos laboratoriais, composta por 4 dentes $(34,35,36$ e 37) presos a uma base de acrílico. Este foi utilizado em todas as moldagens.

As moldagens foram realizadas com moldeiras parciais em aço pré-fabricadas, perfuradas e devidamente adesivadas (Universal Tray Adhesive, Zhermack, Badia Polesine, Itália), seguindo recomendações do fabricante. Após sua aplicação, foi aguardada sua secagem durante dois minutos de forma natural, sem jato de ar.

Para todas as técnicas, o silicone denso foi proporcionado com as colheres dosadoras fornecidas pelo fabricante em partes iguais cada uma das pastas. Segundo recomendações do fabricante, a manipulação do material denso foi realizada por 30 segundos, até obter uma massa de cor homogênea. As luvas, por conterem em sua formulação componentes que alteram as propriedades do PVS, foi manipulado sem elas, com as mãos limpas e secas. O total tempo de polimerização aguardado para esse material foi de 5 minutos e meio. O silicone fluido foi manipulado através de um dispensador-pistola (3M ESPE, Saint Paul, EUA), com auxílio de uma ponta de auto-mistura. Em todas as técnicas ele foi dispensado tanto sobre o material denso no molde quando sobre o modelo piloto. O tempo de polimerização aguardado foi também de 5 minutos e meio.

Foram testadas as seguintes técnicas de moldagem:

\section{T1 - Técnica de 2 passos com recorte}

Inicialmente foi realizada a moldagem com material denso, levando a moldeira carregada, após adesivada, ao modelo piloto, inserindo-a em um único sentido, sem fazer movimentos de báscula. Após polimerização total do material, a moldeira foi removida em movimento único. Então, foi utilizado um instrumento (talhador de madeira) para produzir recortes na região do término do preparo e nas laterais do molde com silicone denso, proporcionando um espaço para escoamento do material fluido, que foi inserido em seguida, levando o molde em posição novamente e aguardando o tempo da reação de polimerização.

\section{T2 - Técnica de 2 passos com alívio de filme PVC (Policloreto} de vinila)

Primeiramente foi realizada uma moldagem com o material denso, conforme descrito anteriormente, porém com uma película de filme PVC entre o silicone e os dentes a serem moldados. Após sua reação de polimerização, a película foi removida e o molde foi preenchido com material fluido e levado novamente ao modelo até a reação de polimerização completa.

\section{T3 - Técnica de 2 passos sem alívio}

O silicone denso foi carregado na moldeira adesivada e levado modelo piloto para a obtenção do primeiro molde. Nenhum recorte ou alívio foi realizado, logo em seguida, foi inserido o material fluido, sobre o molde preliminar e a moldeira foi inserida novamente no modelo piloto, aguardando-se sua polimerização.

\section{T4 - Técnica de 1 passo ou simultânea}

$\mathrm{O}$ material denso foi manipulado e levado à moldeira e ao mesmo tempo, o material fluido foi inserido no modelo e na moldeira, sobre o material denso. O conjunto foi levado ao modelo piloto em movimento único e aguardou-se o tempo da reação de polimerização.

Todas as moldagens foram realizadas pelo mesmo operador treinado. Após a moldagem, todos os moldes passaram por uma análise criteriosa afim de classificá-las quanto à sua qualidade. Apenas as moldagens que não apresentavam nenhum defeito foram utilizadas, totalizando 4 moldagens finais - uma para cada técnica estudada $(\mathrm{n}=1)$.

Apenas após 30 minutos da obtenção dos moldes, os mesmos foram vazados para confecção dos modelos, permitindo a recuperação elástica do material. Para a obtenção dos modelos utilizou-se a proporção sugerida pelo fabricante; $100 \mathrm{~g}$ de gesso tipo IV (Durone, Dentsply, Rio de Janeiro, Brasil), para $19 \mathrm{~mL}$ de água, manipulado manualmente, inserindo o pó à água até este estar completamente aglutinado. $\mathrm{O}$ gesso foi vertido nos moldes usando um vibrador de gesso, evitando a formação de bolhas no 
modelo final. Após 30 minutos, as amostras foram removidas dos moldes, e após 24 horas os modelos finais foram analisados.

Para a análise dos modelos foram marcados 10 pontos correspondentes no modelo piloto e nas amostras. Para que as marcações fossem padronizadas foi confeccionado um guia em acetato em plastificadora manual. O guia foi perfurado nos 10 pontos de referência, e, com seu auxílio, foram feitas as marcações nos modelos obtidos e no modelo piloto (Figura 1).

Tendo como base esses 10 pontos, foram realizadas cinco medidas (M1, M2, M3, M4 e M5) (Figura 2) com paquímetro digital (Nove 54, Curitiba, Brasil), pelo mesmo operador. Cada modelo e cada medida foram mensurados 10 vezes $(n=10)$.

Os valores obtidos das medidas dos modelos foram tabulados e analisados no programa Stat Plus:Mac v.6.2.21 (Analysoft Inc, Atlanta, EUA). Inicialmente os dados foram testados quanto a sua homogeneidade (Teste de Levene) e normalidade (Teste de Kolmogorov-Smirnov). Devido a sua distribuição paramétrica e homogênea, foi utilizado o teste ANOVA com comparações múltiplas pelo teste de Tukey $(\alpha=0,05)$ para comparar as mesmas medidas entre os modelos obtidos pelas diferentes técnicas.

Os modelos foram escaneados em scanner digital (3Shape D800, 3Shape, Copenhague, Dinamarca) para análise em sistema CAD/CAM (Wieland, Zenotec, Pforzheim, Alemanha). As imagens dos modelos obtidos por cada técnica e do piloto foram sobrepostas no sistema a fim de verificar a quantidade de coincidência ou discrepâncias entre elas, como forma de ilustrar as diferenças entre o modelo original e o modelo obtido.

\section{RESULTADOS}

As médias das diferentes medidas obtidas para cada modelo nas diferentes técnicas de moldagem estão apresentadas na figura 3. Todas as técnicas de moldagem apresentaram diferença estatística do modelo piloto em alguma das medidas $(\mathrm{p}<0,05)$. Os valores de desvios-padrão encontrados variaram de 0,04 a 0,15 e por isso, não seriam bem representados no gráfico. As técnicas T1, T2 e T3 apresentaram diferença estatística do piloto em 3 medidas, enquanto a técnica $\mathrm{T} 4$ apresentou diferença estatística do piloto em 4 das 5 medidas, sendo, portanto, a que apresentou maior diferença. Sabendo que o teste estatístico considera como significante qualquer diferença maior que $5 \%$, pode-se dizer que clinicamente, talvez, essas diferenças não sejam significantes.

\section{Análise das imagens}

as imagens do modelo piloto e dos modelos em gesso obtidas através do escaneamento no CAD/CAM, foram analisadas através de sobreposição e estão apresentadas nas figuras 4, 5, 6 e 7. A cor em azul nas imagens é referente ao modelo piloto, e a cor em amarelo é referente ao modelo em gesso. Analisando as imagens, observou-se que em todas as amostras os modelos em gesso aparentam ter alterações em relação ao modelo piloto, confirmando o resultado obtido através das aferições.

As técnicas T1, T2 e T3 não apresentaram alterações dimensionais significativas com todas as imagens apresentando uma mescla das cores (Figuras 4, 5 e 6). Observa-se que em todas as técnicas estudadas ocorre aumento de volume nos modelos, isso pode ser explicado pela expansão do gesso. Dentre os três, o modelo T3 apresentou as maiores diferenças em relação ao modelo original de acordo com as imagens.

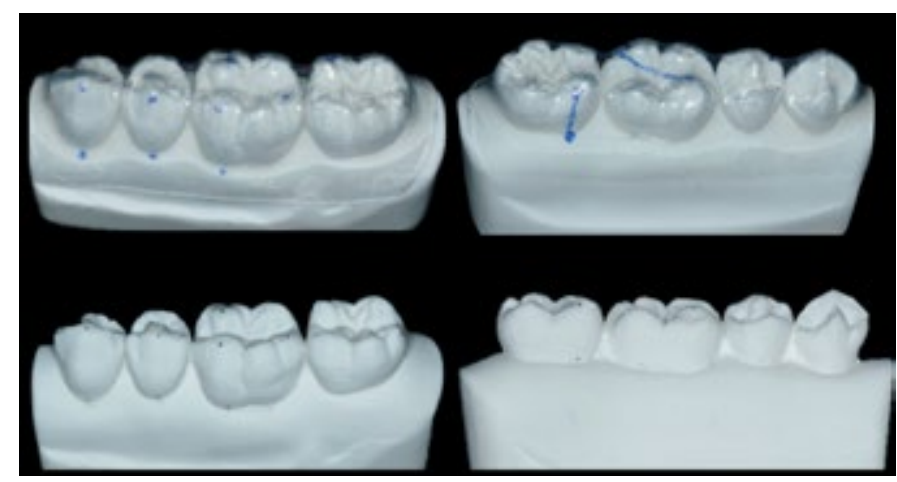

Figura 1 - Marcações dos pontos de mensuração no modelo de gesso com guia em acetato.

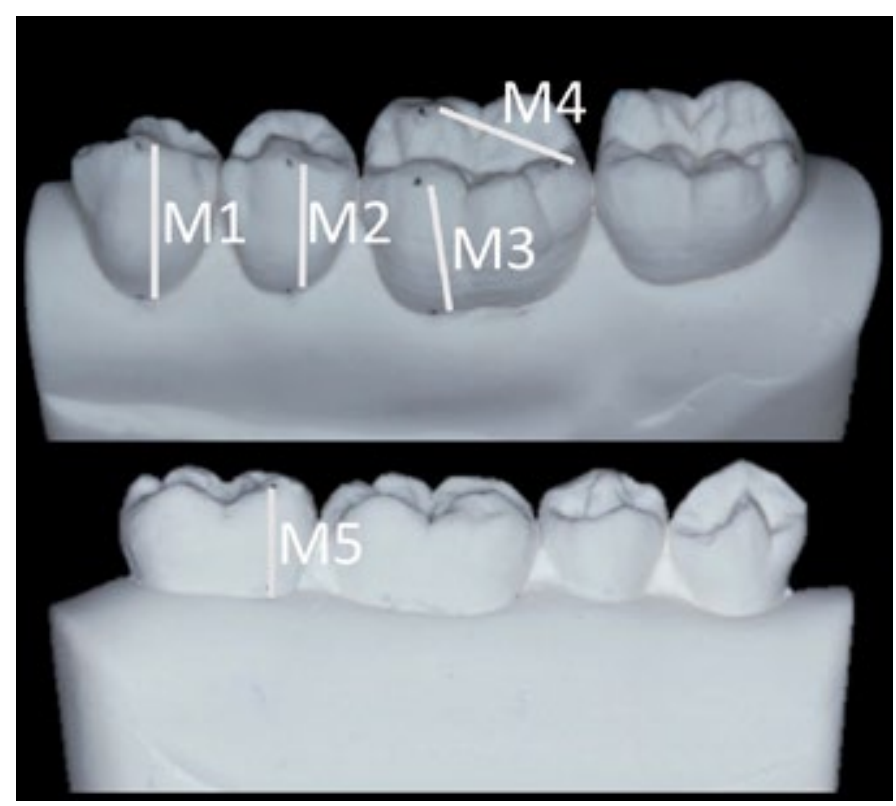

Figura 2 - Pontos já demarcados com caneta hidrocor permanente.

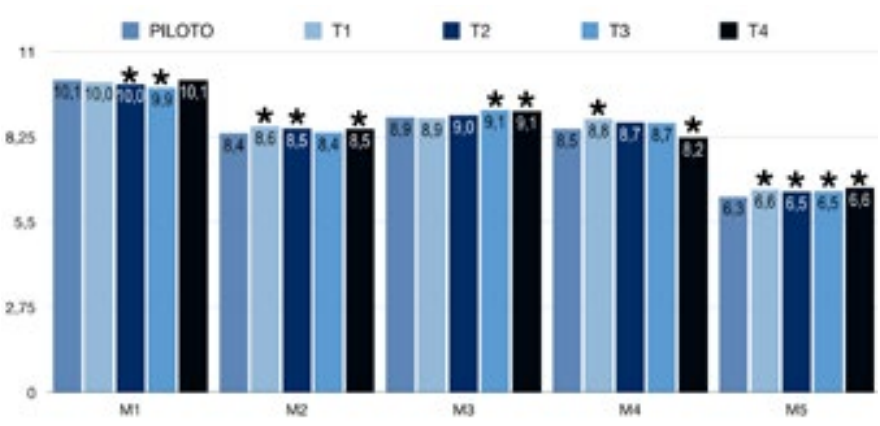

Figura 3 - Médias de cada medida obtida para cada modelo nas diferentes técnicas de moldagem. Valores seguidos pelo asterisco apresentam diferença estatística do piloto $(\mathrm{p}<0,05)$.

Na técnica T4 observa-se que houve alterações dimensionais mais significativas quanto ao tamanho da amostra, além de apresentar na mesial e distal do modelo a presença de repuxo (Figura 7), característica comum em moldes obtidos pela técnica simultânea. Outra característica presente, observando-se a face lingual, (Figura 7B) é o dobramento do material de moldagem próximo ao término, isso ocorreu pelo excesso de força aplicada 
no momento da inserção da moldeira. Não houveram alterações significativas na face oclusal.

\section{DISCUSSÃO}

Vários fatores estão relacionados com a realização de uma boa moldagem, incluindo o material escolhido, a técnica empregada, a alteração dimensional do molde e a moldeira utilizada ${ }^{16,18}$. Diversos estudos demostram as características de cada técnica existente, expondo suas qualidades e dificuldades para a obtenção de resultados melhores ${ }^{16,18}$.

Neste estudo a técnica de um passo foi a que apresentou maiores diferenças do modelo piloto. Estudos demostram que a técnica de um passo é muito utilizada no dia a dia do consultório, já que apresenta como maior vantagem a redução do tempo clínico; mas também apresenta desvantagens, como a diminuição na precisão do molde quando comparada às outras técnicas, em conformidade com os achados desse estudo ${ }^{6,9,18}$. Há também relatos de que podem haver alterações dimensionais maiores nos modelos finais, devido à polimerização dos materiais acontecer em apenas uma etapa ${ }^{17,18}$. Outra característica negativa da técnica de um passo, é que existe uma tendência de o material pesado ser empurrado contra as paredes do preparo. Com isso, o material leve pode ser removido do término do preparo, sendo substituído pelo material pesado, portanto os detalhes não podem ser copiados de maneira satisfatória ${ }^{19,20}$. A técnica de um passo apresenta desafios que podem comprometer a qualidade do molde, um desses desafios é a ocorrência de movimentos de báscula durante a inserção da moldeira, esse movimento pode causar alterações dimensionais além de ocorrer, em muitos casos, o aparecimento de vazios no molde ${ }^{3}$.

A técnica de dois passos sem alívio apresenta bons resultados clínicos, todas as características do preparo são reproduzidas de forma satisfatória, porém estudos mostram que essa técnica pode apresentar reproduções de dimensões maiores que os modelos iniciais ${ }^{6,9}$. Como vantagens, nessa técnica apenas o material fluido copia o término do preparo, e como a técnica é realizada em duas etapas, a contração de polimerização é inexistente ${ }^{17,18}$.
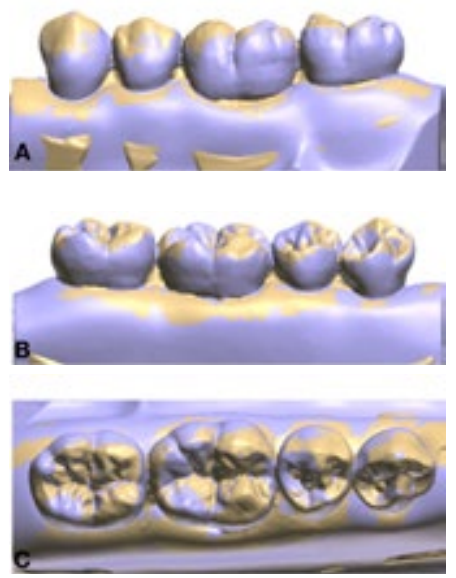

Figura 4 - Técnica T1: A) Face vestibular; B) Face lingual; C) Face oclusal.
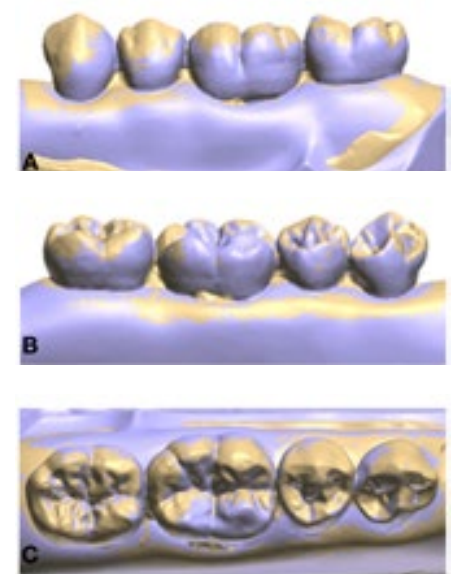

Figura 5 - Técnica T2: A) Face vestibular; B) Face lingual; C) Face oclusal.
O estudo de Kumari e Nandeeshwar ${ }^{16}$ (2015) mostra em seus resultados que a técnica de moldagem de dois passos sem alívio quando comparada a técnica de um passo apresenta resultados melhores, em termos de precisão na cópia dos detalhes e estabilidade dimensional. Segundo Bansal ${ }^{6}$ (2010), o resultado de seu estudo mostrou que não há diferenças estatísticas quando comparadas as técnicas de um passo e de dois passos sem alívio - não há diferenças significativas que influenciem no resultado final da prótese. Nesse estudo, a técnica de dois passos sem alívio foi comparável às técnicas com alívio nas comparações das medidas dos modelos, porém em CAD/CAM foi possível observar um pouco mais de diferença entre o modelo piloto e o final quando a mesma foi realizada.

A técnica de dois passos com recorte ou alívio é descrita na literatura como sendo um dos métodos mais eficientes ${ }^{3,6,9,17,18}$. Estudos mostram que a técnica de 2 passos com alívio apresenta resultados mais satisfatórios, devido ao espaço criado na moldagem preliminar, já que o alívio limita a quantidade de material fluido, diminuindo o risco de ocorrerem alterações dimensionais $^{6,9,18}$. Alguns estudos descrevem que idealmente o alívio no molde deve ser de $2 \mathrm{~mm}$ para uma melhor qualidade do mol$\mathrm{de}^{9,18}$, porém, clinicamente, se torna inviável essa mensuração e padronização da quantidade de material removido ou aliviado. Por se tratar de um procedimento em duas etapas e por ser difícil promover uma padronização da confecção do recorte ou alívio, torna-se difícil sua reprodução por diferentes profissionais ou até pela mesma pessoa em diferentes momentos.

Em conformidade com a literatura, a técnica de dois passos com alívio apresentou os melhores resultados nesse estudo, seja com recorte ou com filme PVC. Os dois métodos abordam a utilização de artifícios para criar um espaço que será preenchido pelo material fluido, diminuindo a quantidade do mesmo e favorecendo sua compressão sobre os tecidos. Apesar de oferecer o mesmo propósito, a reprodutibilidade do recorte torna-se difícil, e também não é possível padronizar a quantidade de material removido. Porém, acredita-se que o uso do filme PVC para alívio, apesar de não relatado na literatura, é mais fácil de ser reproduzido por diferentes profissionais, em diferentes momentos e deve ser mais estudado a fim de validar seu uso.
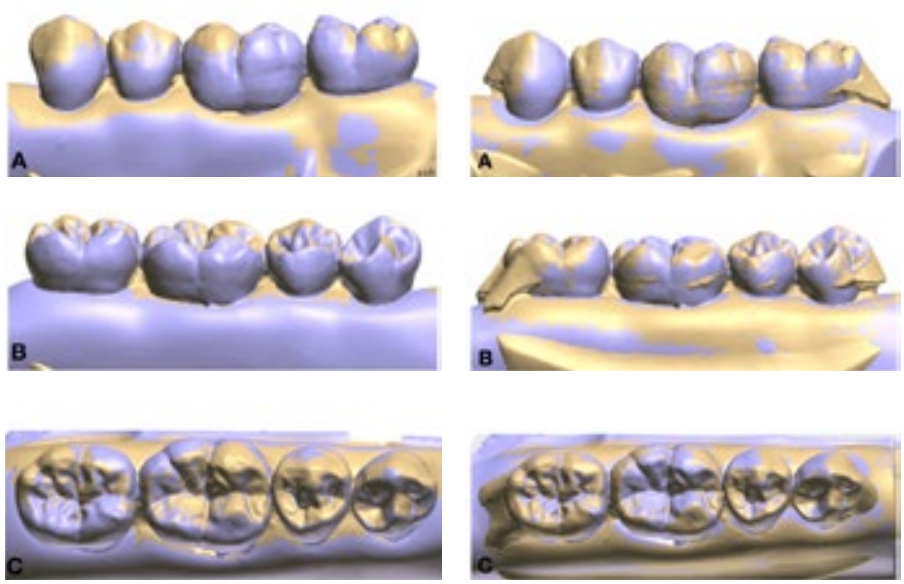

Figura 6 - Técnica T3: A) Face vestibular; B) Face lingual; C) Face oclusal.
Figura 7 - Técnica T4: A) Face vestibular, observar o repuxo nas faces proximais; B) Face lingual, observar dobramento do material próximo à região cervical; C) Face oclusal. 


\section{CONCLUSÃO}

Dentro das limitações desse estudo, pode-se concluir que as diferentes técnicas de moldagem afetam a precisão dimensional dos modelos. Analisando as técnicas estudadas conclui-se que:

1. Todas as técnicas estudadas geraram alterações dimensionais nos modelos obtidos, em especial a simultânea, que apresentou diferença estatística em mais pontos que os outros modelos estudados;

2. As imagens analisadas pelo sistema CAD/CAM confirmam essa alteração dimensional, principalmente nas técnicas dois passos sem alívio e simultânea;

3. As diferenças estatísticas podem não ter significância clínica, porém observa-se que a depender da técnica empregada, o modelo obtido pode apresentar mais ou menos alterações dimensionais.

\section{REFERÊNCIAS}

01. Sinhoreti MAC, Vitti RP, Mendonça MJ, Consani RLX, CorrerSobrinho L. Estudo da precisão dimensional de modelos de gesso confeccionados com diferentes técnicas e materiais de moldagem elastoméricos. Rev Facul Odon. 2010; 15 (2): 139-44.

02. Raigrodski AJ, Dogan S, Mancl LA, Heindl H. A clinical comparison of two vinyl polysiloxane impression materials using the one-step technique. J Prosthet Dent. 2009; 102 (3): 179-86.

03. Agra CM, Ramalho GC, Anfe TEA, Nagase DY. Moldagem protética em dois passos - detalhes técnicos. J of Biodent and Biomat. 2014; 4 (1): $36-50$

04. Samet N, Shohat M, Livny A, Weiss EI. A clinical evaluation of fixed partial denture impressions. J Prosthet Dent. 2005; 94 (2): 112-7.

05. Soares PV, Santos-Filho PC, Martins LR, Soares CJ. Influence of restorative technique on the biomechanical behavior of endodontically treated maxillary premolars. Part I: fracture resistance and fracture mode. J Prosthet Dent. 2008; 99 (1): 30-7.

06. Bansal PK. Comparison of dimensional accuracy using two elastomeric impression materials in fixed prosthodontics. Pakist Oral \& Dent J. 2010; 30 (2): 537-44.

07. Starling J. Avaliação da alteração dimensional de siliconas de reação por condensação e adição, em função dos modelos de gesso obtidos em diferentes tempos de armazenagem dos seus moldes [Dissertação de Mestrado]. Belo Horizonte: Faculdade de Odontologia da UFMG; 2003.
08. Zanetti GR, Peçanha MM, Frizzera F, Monteiro-Júnior C. Moldagem de precisão em prótese fixa. Rev Dental Press Estét. 2011; 8 (1): 46-54.

09. Dias DRCM, Barroso FT, Heleno GLG, Simões NM, Ramos BC, Manzi FR et al. Dimensão do alívio em técnica de dupla moldagem: uma revisão de literatura. Arq Bras Odont. 2016: 12 (1): 18-23.

10. Shen C. Materiais de moldagem In: Phillips AK. Materiais Dentários. 11. ed. Rio de Janeiro: Elsevier; 2005. p. 193-238.

11. Mezzomo E, Lopes LA, Suzuki RM. Materiais e Técnicas de Moldagens. In: Mezzomo E, Suzuki RM. Reabilitação oral contemporânea. São Paulo: Santos; 2006. p. 637-709.

12. Beier US, Grunert I, Kulmer S, Dumfahrt H. Quality of impressions using hydrophilic polyvinyl siloxane in a clinical study of 249 patients. Int J Prosthodont. 2007; 20 (3): 270-4.

13. Basapogu S, Pilla A, Pathipaka S. Dimensional Accuracy of Hydrophilic and Hydrophobic VPS Impression Materials Using Different Impression Techniques - An Invitro Study. J Clin Diagn Res. 2016; 10 (2): ZC56-9.

14. Dogan S, Schwedhelm ER, Heindl H, Mancl L, Raigrodski AJ. Clinical efficacy of polyvinyl siloxane impression materials using the one-step two-viscosity impression technique. J Prosthet Dent. 2015; 114 (2): 217-22.

15. Hoyos A. Influence of tray rigidity and material thickness on accuracy of polyvinyl siloxane impressions [Dissertação de Mestrado]. Gainsville: Faculdade de Odontologia da University of Flórida; 2006.

16. Kumari N, Nandeeshwar DB. The dimensional accuracy of polyvinyl siloxane impression materials using two different impression techniques: An in vitro study. J Indian Prosthodont Soc. 2015; 15 (3): 211-7.

17. Franco EB, Cunha LF, Herrera FS, Benetti AR. Accuracy of SingleStep versus 2-Step Double-Mix Impression Technique. ISRN Dent. 2011; 2011: 341546.

18. Caputi S, Varvara G. Dimensional accuracy of resultant casts made by a monophase, one-step and two-step, and a novel two-step putty/ light-body impression technique: an in vitro study. J Prosthet Dent. 2008; 99 (4): 274-81.

19. Chee WW, Donovan TE. Fine detail reproduction of very high viscosity poly(vinyl siloxane) impression materials. Int J Prosthodont. 1989; 2 (4): 368-70.

20. Morgano SM, Milot P, Ducharme P, Rose L. Ability of various impression materials to produce duplicate dies from successive impressions. J Prosthet Dent. 1995; 73 (4): 333-40.

\section{ABSTRACT}

Objetives: To compare different impression techniques with addition silicone, by means of copy accuracy through manual measurements and overlaping in CAD/CAM. Material and methods: Four impressions of a same pilot model were carried out with silicone addition in different techniques: T1 - two steps with cut-back; T2 - two steps with PVC film relief; T3 - two steps without relief; and T4 - one step. The accuracy of the 4 different impression techniques was assessed by measuring 5 dimensions on stone casts poured from the impressions of the pilot model. Another analysis was carried out in CAD/CAM using the overlaping of the casts and the pilot model, in order to verify dimensional similarities and differences between them.
The values obtained were submitted to ANOVA and Tukey test, for multiple comparisons $(\alpha=0.05)$. Results: According to the measures done, the technique that generated the greatest difference from the pilot model was T4, with no difference among the other techniques. In CAD/CAM analysis, techniques T4 and $\mathrm{T} 3$ presented more differences from the original model. Conclusions: All studied techniques presented dimensional changes, mainly the 1 step and the 2 step without relief, in both methods, measures and CAD/CAM analysis.

Keywords: Dental Impression Materials, Dental Impression Technique, Silicone Elastomers, Anatomic Models. 


\section{AUTOR PARA CORRESPONDÊNCIA}

Ana Paula Rodrigues de Magalhães

Endereço: Av. Itália, 1184 - Jardim Europa

CEP: 74325-110

Goiânia - GO, Brasil.

Telefone: 556232363100

E-mail: anapaulardm@gmail.com 